\title{
The curriculum, the macro design and the micro design, and the curriculum transposition ${ }^{1}$
}

Pier Giuseppe Rossi ${ }^{2}$

\begin{abstract}
The didactical design is organized in different documents that are peculiar in the different countries. In Italy there id the school curriculum starting from the national guidelines for the pre-school, primary and middle school, and from the regulations for the high school. Then many teachers create yearly design documents for each discipline collaborating, at times, with colleague of parallel classes. In this article, such yearly documents will be called "macro design", while the design of the single work sessions (from 1 to 8 hours in class) will be called "micro design". The complexity, finally, requires that macro and micro design are transponed and it is needed that a design artefact can be shared in class with students starting processes of co-design.

The article analyses the role and the structure of the school curriculum in the current context, the relationships between the macro and micro design and, finally, the need of a transposition of the design artefacts to share the design with students.

The article "the curriculum", present in the same issue, is the logic premise of the present contribution
\end{abstract}

Keywords: curriculum, macro and micro-design, visible design.

\section{Premessa}

Quali possano essere gli artefatti progettuali di cui la didattica necessita?

L'analisi di Stenhouse già individuava due possibili strutture per il curricolo: una prima è costituita da linee guida per la costruzione di percorsi, la seconda sono i percorsi stessi. Ugualmente Joannert (2001) e Joannert e de Brorght (2009) distinguono "between the concepts of 'curriculum' and

${ }^{1}$ Il presente contributo è fortemente connesso al contributo: Il Curricolo, sempre nel presente numero della rivista.

${ }^{2}$ Pier Giuseppe Rossi is Full Professor on Didactics and Special Pedagogy, Department of Education, Cultural Heritage and Tourism, University of Macerata. 
'programme of study"'. In realtà riteniamo che le due strutture possano essere contemporaneamente presenti nell'agire didattico, spesso effettuate da attori diversi e relative a destinatari differenti. Va precisato, comunque, che la presenza di un artefatto che descriva la successione delle attività, ad esempio in una didattica per competenze, non è precisamente un programme of study.

Per Curricolo di istituto intendiamo, nel contesto italiano, una serie di linee guida generative in base alle quali il docente costruisce una macro e una micro progettazione. Tale visione è coerente con quanto prescritto dalle indicazioni nazionali.

Il curricolo d'istituto è espressione della libertà di insegnamento e dell'autonomia scolastica e allo stesso tempo, esplicita le scelte della comunità scolastica e l'identità dell'istituto. La costruzione del curricolo è il processo attraverso il quale si sviluppano e organizzano la ricerca e l'innovazione scolastica.

A partire dal curricolo d'istituto i docenti individuano le esperienze di apprendimento più efficaci, le scelte didattiche più significative, le strategie più idonee, con attenzione all'integrazione tra discipline e alla loro possibile aggregazione in aree (IN 2012, 17).

Gli insegnanti, singolarmente o per classi parallele, articolano i propri percorsi annuali - la macro-progettazione - nella quale, in base alle Indicazioni nazionali, alle linee guida presenti nel Curricolo di istituto, alle caratteristiche della classe, al contesto in cui la scuola è inserita e alla propria filosofia educativa disegnano il percorso che intendono realizzare.

In base al curricolo e alla macro-progettazione viene poi prodotta, anche in questo caso in modo non meccanico, la micro-progettazione, ovvero la pianificazione delle sessioni di lavoro quotidiane o settimanali.

Vi è infine un terzo livello, quello del curricolo trasposto (Joannert, 2011; Rossi, 2016) o del visible design, che prevede la costruzione di artefatti che visualizzino la macro e la micro-progettazione in classe in un formato grafico e linguistico adatto alla classe. Sono gli artefatti progettuali da condividere con gli studenti, come si vedrà successivamente, utili per l'orientamento e la coprogettazione con gli studenti.

\section{II curricolo come sapere-strumento}

Quando una logica era predominante nel contesto sociale e scolastico, il curricolo esplicitava e articolava tale logica nelle specifiche discipline. Tali logiche erano principalmente due: quella della successione temporale, presente in discipline quali le letterature, la storia, la filosofia; quella della struttura gerarchica dal particolare al generale o dal semplice al complesso, presente 
in discipline quali la geometria, la grammatica, la fisica e la chimica. Anche nel curricolo per obiettivi il concetto stesso di obiettivo e la sua struttura a matrioska erano il riferimento prevalente per la progettazione. La presenza pervasiva di tale logiche rendeva spesso inutile una esplicitazione; erano nel vissuto di docenti e studenti come presenza assoluta e ovvia e sembravano non frutto di una scelta. Inoltre tali strutture erano reificate dall'indice del libro di testo.

L'assenza oggi di una logica predominante e la presenza di più logiche/ processi, tra loro autonomi anche se interdipendenti, assegna al docente il compito di costruire in contesto e per ogni disciplina una rete di senso che connetta e renda coerente un percorso in cui le varie logiche e i "materiali" dialogano, siano essi le competenze, le esperienze e conoscenze dello studente, e il sapere sapiente. In tale direzione il curricolo diviene un sapere-strumento che supporta il singolo docente nella costruzione di una tale rete di senso.

Il curricolo suggerisce le modalità con cui aggregare i frammenti ed è generativo in quanto suggerisce possibili processi con cui costruire in contesto puzzle significativi.

Quali elementi potrebbero costituire un Curricolo di istituto?

Sicuramente un Curricolo deve individuare:

Le scelte educative da condividere nell'istituzione.

Le indicazioni e gli strumenti per aggregare.

Le prassi da condividere nella scuola.

\section{Scelte educative da condividere nell'istituzione}

Il curricolo fornisce alcuni riferimenti educativi sul ruolo dell'istituzione scolastica nello specifico contesto, indicazioni che caratterizzano lo stile e la cultura di un dato istituto. Ad esempio potrebbe suggerire come interpretare la centralità dello studente, come favorirne un ruolo attivo, ma solo se si colgono aspetti caratterizzanti, anche se in uno specifico intervallo temporale. Non ha senso che siano esplicitati in ogni curricolo gli elementi presenti nelle Indicazioni o già condivisi e patrimonio di una cultura scolastica più ampia $\mathrm{e}$ generalizzata, che oltrepassa le mura del singolo istituo.

Ugualmente il curricolo potrebbe esplicitare la politica che la scuola intende sviluppare nel territorio, specialmente se da essa derivano progetti e attività didattiche. La scuola si connette con il contesto sociale e culturale in cui è inserita e valorizza specifiche risorse e valori in esso presenti. Resta sottinteso che il curricolo dialoga con il PTOF nel quale sono esplicitati elementi connessi alla mission dell'Istituto. 


\section{Le indicazioni e gli strumenti per aggregare}

Il curricolo suggerisce poi degli strumenti che come dei filtri permettono di osservare i processi in atto o di predisporne dei nuovi facendo emergere quei fili rossi che aggregano frammenti diversi, differenti attività, differenti processi e fanno emergere il senso dei processi stessi.

\section{Le competenze chiave europee}

Un primo filtro per aggregare sono le competenze chiave, oggi fondamentali per la formazione del cittadino di domani, un cittadino che sappia affrontare l'imprevisto e sappia lavorare sulla propria identità, professionale e personale, progettando e inventando il proprio futuro.

In realtà le competenze possono essere viste in differenti prospettive:

- come traguardi, e in questo senso alla stregua di obiettivi presentano dei modi di essere cristallizzati;

- come proposte operative con cui risolvere delle famiglie di situazioni problematiche;

- come schemi concettuali con cui osservare le attività in classe e in tal senso sono uno strumento strutturante, in quanto propongono specifici punti di vista con cui rilevare elementi dalle pratiche presenti, e strutturato, in quanto nell'attività il soggetto, che riprogetta se stesso (logica costruttiva) mentre risolve un problema (logica produttiva), organizza anche il suo modo di essere competente. In altri termini l'essere competente del soggetto prende forma nell'azione.

Il curricolo, quando utilizza la competenza chiave come strumento di aggregazione, assume questa terza prospettiva ovvero utilizza le competenze come schema concettuale per cercare dei fili rossi che attraversano le attività e le connettono le une con le altre. Poiché le competenze chiave sono già presenti nelle Indicazioni nazionali e nelle Linee guida europee, nel curricolo è opportuno solo indicare quella o quelle competenze su cui si vuole concentrare in un certo intervallo temporale l'attenzione, ben sapendo che le competenze sono un corpo unico e che in molti processi sono tra loro connesse e intrecciate.

\section{I nuclei fondanti}

I nuclei fondanti sono concetti che ritornano ricorsivamente nei percorsi disciplinari, anche se con formalismi diversi e con definizioni diverse ${ }^{3}$.

\footnotetext{
${ }^{3}$ In ambito della didattica della matematica un concetto simile a nucleo fondante è quello di campo concettuale.
} 
Danno continuità verticale al sapere disciplinare e orientano gli studenti. Non sono i contenuti irrinunciabili delle varie discipline, né contenuti che ritornano ciclicamente. Sono "categorie" e logiche presenti in ogni disciplina che ricorsivamente ritornano nel percorso verticale. Alcuni esempi possono chiarire il significato di nucleo fondante. Nella didattica della matematica sono nuclei fondanti i concetti di numero, di relazione, di trasformazione; in storia i concetti di cambiamento e persistenza, di globalizzazione, di rivoluzione. Il concetto di relazione in matematica si presenta fin dalla scuola dell'infanzia quando si raggruppano oggetti in funzione di specifiche caratteristiche. Il concetto di relazione ritorna nei primi anni della scuola primaria con le operazioni matematiche e, poi, in particolari categorie di problemi come quelli sul peso-netto, peso-lordo e tara, o sulla spesa, guadagno e ricavo. Nella scuola media di primo grado l'introduzione dell'algebra fa riemergere il concetto di relazione e, se non ben connesso con un percorso verticale, l'algebra rappresenta un ostacolo insormontabile per molti ragazzi perché non si coglie la continuità con il percorso precedente. Vedere la relazione come nucleo fondante abitua lo studente a una prospettiva e a un linguaggio che gli fornisce una chiave di lettura nei momenti in cui vengono introdotti nuovi elementi. Va anche sottolineato che il concetto di relazione oltrepassa l'ambito disciplinare e viene utilizzato in modo significativo, anche se con curvature diverse, in discipline quali scienze, fisica e alcune materie umanistiche. Qualcuno potrebbe obiettare sulla utilità di inserire i nuclei fondanti in un Curricolo di istituto in quanto dovrebbero essere prodotti dalla ricerca disciplinare e indipendente dai contesti. Si ritiene invece che l'individuazione dei nuclei concettuali non è facilmente circoscrivibile e molteplici potrebbero essere i nuclei di una disciplina anche a partire da approcci epistemologici diversi. È possibile, pertanto, sceglierne alcuni nei singoli istituti su cui fondare dei percorsi verticali, focalizzare la formazione in un dato periodo e costruire linguaggi condivisi ${ }^{4}$.

\section{Le operazioni cognitive}

Le operazioni cognitive si deducono dai traguardi di competenza e dalla struttura della disciplina e caratterizzano specifiche attività anche in funzione delle consegne con cui vengono proposte. Sono connesse alle competenze disciplinari e individuano i processi che l'alunno mette in atto nell'agire disciplinare. Permettono al docente di predisporre il dispositivo/compito

\footnotetext{
${ }^{4}$ Un altro contributo di questo numero monografico approfondisce senso e utilizzo dei nuclei fondanti.
} 
prevedendo le attività che deve compiere lo studente sui saperi. Sono esempi di operazioni cognitive in italiano effettuare inferenze sui significati lessicali, riorganizzare il testo in base alla temporalità o altri indicatori, utilizzare il registro linguistico adeguato in relazione al contesto; in storia: mettere in relazione fatti e concetti con i contesti, produrre testi storiografici verbali e non verbali, selezionare/ricercare informazioni di tipo geo-storico, osservare elementi significativi dei contesti. Gli operatori cognitivi svolgono un ruolo di aggregazione in quanto spesso possono rappresentare un ponte tra i saperi disciplinari e le competenze e quindi garantiscono la presenza di una rete di senso. Inserire le operazioni cognitive nel Curricolo di istituto permette di identificare processi sui quali si intende focalizzare l'attenzione in una certa fase dello sviluppo degli studenti.

\section{Alcune prassi da condividere}

Un ulteriore campo, presente nel curricolo e utile per creare una rete tra $\mathrm{i}$ percorsi di più docenti nella classe e tra più classi in verticale, è l'indicazione di alcune prassi e processi su cui la scuola ritiene utile focalizzare l'attenzione in un dato intervallo temporale. Tali prassi sono fortemente suggerite per concentrare il lavoro su specifiche strategie o attività. Se vari docenti utilizzano una specifica strategia, la comunità dell'istituto può avviare percorsi di formazione mirati e confrontare i risultati verificandone l'impatto sugli apprendimenti e l'efficacia educativa. La scelta di lavorare per laboratori, di privilegiare il lavoro di gruppo, di adottare EAS (Rivoltella, 2013), di svolgere alcuni processi va in questa direzione. Inserirlo nel curricolo non significa che quelle divengono le uniche pratiche didattiche possibili, ma che una data prassi sarà seguita con particolare attenzione e proposta da più docenti anche in modo sperimentale, ben sapendo che una prassi è efficace se condivisa in una classe da più docenti in modo tale che gli studenti se ne approprino e vi partecipino in modo consapevole.

Un'ulteriore prassi da generalizzare per favorire una cultura condivisa nell'istituzione in questa fase storica è relativa alle modalità con cui certificare le competenze. La certificazione delle competenze è un percorso richiesto alla scuola da indicazioni ministeriali. È una prassi nuova che richiede modalità operative da sperimentare e che pertanto è utile condividere. Ritenere che sia utile esplicitare nel curricolo una tale indicazione implica che alcuni docenti ne discutano prima in piccolo, poi in grande gruppo, costruendo una cultura di istituto da validare in una prima fase in modo che possa poi essere messa a regime e diventare un habitus consolidato.

Suggerire alcune prassi, più che atto prescrittivo, impatta sul processo identitario dell'istituto e, connesso a esso, sul processo di crescita professionale 
del corpo insegnante in quanto permette di attivare politiche formative e organizzative mirate e condivise. In altri termini la struttura di un Curricolo di istituto rappresenta un documento identitario e programmatico che genera direttamente e indirettamente prassi sia micro, ovvero l'azione didattica dei singoli docenti, sia macro, ovvero le linee di coordinamento dell'intero istituto sul piano della formazione e della progettualità con il territorio.

\section{La costruzione del Curricolo di istituto}

Negli ultimi anni vari istituti, per adempiere a un compito richiesto dalle Indicazioni nazionali e dalla normativa vigente, hanno costruito il Curricolo di istituto. $\mathrm{Ci}$ si potrebbe chiedere per quale motivo non venisse fornito dall'alto alle scuole un modello. La risposta è in parte nei paragrafi precedenti: un curricolo va costruito in funzione delle esigenze del contesto socio-culturale ed è connesso alla cultura che caratterizza un dato istituto. Questa è solo una delle motivazioni. Durante processo di costruzione i docenti prendono consapevolezza dei sistemi e dei problemi interni ed esterni alla scuola e avviano processi di crescita personale e professionale.

I prodotti realizzati in questi anni non sono sempre e del tutto soddisfacenti, anche per coloro che li hanno realizzati. E questo era inevitabile in quanto chi stava costruendo il curricolo, stava costruendo contemporaneamente la propria competenza e la propria capacità di costruirlo. Si costruiva il prodotto e la professionalità necessaria a realizzarlo.

Indipendentemente dal risultato, però, tutti coloro che hanno partecipato alla costruzione del curricolo affermano che il lavoro è stato molto importante per acquisire una maggiore consapevolezza dei problemi connessi ai processi formativi e al contesto. Inoltre la costruzione di un curricolo ha creato un gruppo di lavoro coeso e capace di sviluppare un sapere in contesto e ha fatto emergere una cultura condivisa, a iniziare dai significati attribuiti ad alcune parole chiave del linguaggio pedagogico-didattico. Gli stessi insegnanti, però, sottolineano anche come tale benefici effetti non sempre sono stati condivisi con l'intero corpo dell'istituto. I docenti che non hanno partecipato alle attività di scrittura del curricolo vedono l'artefatto come un oggetto burocratico di scarsa utilità nella prassi quotidiana. Un tale giudizio è in parte condivisibile in quanto molti dei curricoli prodotti riflettono vecchie impostazioni e presentano una sequenza di obiettivi e competenze che divengono o una struttura troppo rigida da mettere in atto o un corpo di indicazioni di nessuna o scarsa utilità nella prassi quotidiana. Forse il curricolo dovrebbe esplicitare anche le modalità con cui dovrebbe essere condiviso all'interno dell'istituzione. Realizzarlo in piccolo gruppo non equivale 
all'assenza di un processo democratico di condivisione che dovrebbe essere presente sia nel momento iniziale dell'impostazione, sia nel momento finale della validazione. Dalle interviste ${ }^{5}$ a docenti è emerso che la principale critica ai Curricoli di istituto elaborati è relativa allo scarso impatto che essi hanno sulla progettazione e sulle attività di classe. Come ben evidenzia Laurillard la maggioranza dei Curricoli consiste in un elenco di obiettivi (spesso chiamati competenze) mentre forse l'attenzione dovrebbe essere rivolta al ciclo goalknowledge-action-feedback-modulation e alla relazione/allineamento tra learning outcomes $\longleftrightarrow \longrightarrow$ teaching-learning activity $\longleftrightarrow \longrightarrow$ assessment che meglio descrive ciò che è appreso e come (Laurillard, 2012, 96). Un'altra critica è che spesso nei curricoli e nelle pianificazioni non si ritrova la ricchezza del lavoro e della progettualità dei docenti, come se il processo di progettazione nel "nobilizzarla" ne facesse perdere il "sapore".

In effetti l'esame dei molti curricoli di istituto presenti in rete ha permesso di cogliere delle strutture rigide e difficilmente utili per i docenti quando vanno poi a pianificare i loro percorsi annuali e quotidiani. La maggioranza dei curricoli presenti in rete e da noi esaminati, e questo è l'elemento che maggiormente ci ha fatto riflettere, è strutturata in tabelle che rispecchiano la logica lineare dei vecchi curricoli per obiettivi, anche se gli obiettivi sono stati sostituiti, spesso solo nominalmente, dalle competenze.

Si riportano alcuni esempi per concretizzare quanto detto. Nel curricolo di italiano dell'esempio $1^{6}$ la tabella è composta dalle seguenti colonne: microcompetenze, metodologia, contenuti e verifica. Tra le micro-competenze si legge: "• Saper individuare luoghi, personaggi, eventi (rapporto causa-effetto) in un testo ascoltato/letto, - Saper raccontare riferendo personaggi, luoghi, tempi in testi orali e scritti. - Saper raccontare esperienze personali in testi orali e scritti rispettando rapporto causa-effetto" e, più avanti, "Saper costruire mappe strutturali", anche se tutte le voci hanno la stessa struttura e logica. A una prima lettura sembrano obiettivi più che competenze. Inoltre nella colonna "Metodologie" si legge: "• Conversazioni libere e guidate. • Narrazione con uso di immagini e senza. • Lettura e analisi collettiva, individuale di testi. • Individuazione di schemi narrativi. • Utilizzo di schemi per la produzione. • Manipolazione di testi”. Se nella macro-progettazione si indicano le modalità operative della micro, l'assenza di contesto porta a fornire inutili elenchi nei quali essendo tutto presente diviene scarso il supporto quando si passa alla

\footnotetext{
${ }^{5}$ I dati e le interviste sono stati ricavati in incontri con docenti delle Marche e del Friuli Venezia Giulia e da indagini on online che hanno coinvolto globalmente circa 450. Sono in pubblicazione dei materiali di ricerca relativi agli stessi temi.

${ }^{6} \mathrm{http} / / /$ circolodidatticocaluso.it/dcllwpmt14/wp-content/uploads/2015/03/Progr_cicli_ ITALIANO.pdf.
} 
micro-progettazione. In altri curricoli è presente la colonna valutazione in cui sono elencate molte metodologie e anche in questo caso l'inutilità è palese.

Nell'esempio 2 sempre a proposito del curricolo di italiano la struttura prevede quattro blocchi: Ascolto e parlato, Lettura, Scrittura, Riflessione sulla lingua, che nelle classi dalla terza in poi si articolano in Arricchimento lessicale e Approfondimento grammaticale e sintattico. Per ogni blocco è presente una tabella con tre colonne: Traguardi per lo sviluppo delle competenze al termine della classe, Abilità, Conoscenze. Nella colonna Traguardi per la classe terza e nel blocco grammatica si trova: "Riconosce se una frase è o no completa, costituita cioè dagli elementi essenziali (soggetto, verbo, complementi necessari" e tra le abilità: "Compiere semplici osservazioni su testi e discorsi per rilevarne alcune regolarità".

Nell'esempio 3, interessante in quanto presenta differenti livelli (la matrice progettuale unitaria $^{7}$ e il curricolo verticale per discipline ${ }^{8}$ ), la tabella delle discipline è strutturata in 2 colonne per l'infanzia: obiettivi di apprendimento, traguardi delle competenze, e tre colonne per la primaria: conoscenze, abilità, traguardi delle competenze.

Nell'esempio $4^{9}$ un esempio di macro-competenze dell'asse linguistico per la scuola primaria risulta: "Utilizzare in modo appropriato i diversi linguaggi: verbale, iconico, sonoro, motorio". Il curricolo si esaurisce nelle macrocompetenze.

L'esempio $5^{10}$ ha la struttura di una tabella con tre colonne: "Traguardi per lo sviluppo della competenza (dalle Indicazioni Nazionali), Obiettivi di apprendimento (dalle Indicazioni Nazionali) Indicatori di competenza per la valutazione (stabiliti dal Collegio docenti)". Tra gli indicatori di competenza per la voce "ascoltare e parlare" si trova: "• Mantiene l'attenzione e sa cogliere gli elementi essenziali di una conversazione. - Interviene nella conversazione in modo pertinente. - Coglie gli elementi principali e secondari di una narrazione".

Il curricolo $6^{11}$ ha una struttura diversa dalle altre. È un testo narrativo che esplicita i progetti della scuola e le relazioni con il territorio.

Gli esempi riportati, i primi che emergono con una ricerca effettuata con il motore di ricerca Google, non pretendono di essere esaustivi o di fornire una visione completa dello stato dell'arte. Anche se l'analisi è limitata, sembrano, però, esserci alcune costanti: la struttura per tabelle che veicola una logica

\footnotetext{
${ }^{7}$ https://www.icbisuschio.gov.it/wp-content/uploads/2012/11/MPU_0910.pdf.

${ }^{8} \mathrm{https}: / / \mathrm{www}$. icbisuschio.gov.it/wp-content/uploads/2012/11/italiano.pdf.

${ }^{9}$ http://icfavria.gov.it/wp-content/uploads/sites/155/MACRO_Competenze.pdf.

${ }_{10} \mathrm{http} / / / \mathrm{ww}$ w.icfabricadiroma.gov.it/attachments/article/908/Curricolo\%20verticale\%20 parte\%201.pdf.

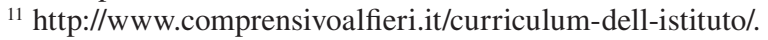


lineare, l'obiettivo o la competenza come elementi base su cui strutturare il curricolo, la presenza di colonne che descrivono caratteristiche tipiche della micro-progettazione (attività e verifiche) e che proprio per questo risultano elenchi omnicomprensivi e generici di nessun aiuto per le attività micro. Il dubbio che vorremmo porre è se la sola indicazione degli obiettivi/competenze possa essere sufficiente compilare un curricolo e essere alla base della pianificazione dei docenti.

La struttura di Curricolo di istituto, presentata nei paragrafi precedenti, non segue questi modelli e adotta una logica narrativa e generativa che dovrebbe supportare la predisposizione delle attività didattiche e il docente nella progettazione. Il concetto di generatività va esplicitato. Il significato assegnato in questa sede al termine "generare" indica non tanto la presenza nel curricolo delle proposte da inserire nella macro e micro progettazione, quanto la presenza di idee e concetti chiave con cui costruire poi le progettazioni e, poi, per verificare la coerenza e la completezza dello stesso. Ad esempio l'indicazione di alcuni nuclei fondanti non impone specifici contenuti o specifiche attività, ma permette di comprendere se le attività pensate hanno elementi in comune e sono connesse ai nodi epistemologici fondanti delle singole discipline.

Un'ultima osservazione. Il curricolo proposto dovrebbe anche essere leggero e flessibile, ovvero richiedere un lavoro limitato nel tempo ed essere facilmente modificabile nel tempo. Strutture molto laboriose, apparentemente complesse e, spesso, sono spesso di difficile validazione e soprattutto raramente sono curvate sulle necessità del contesto in cui viene adottato.

\section{Macro e micro progettazione}

La centralità dei contesti e la logica del Non-uno, ovvero la co-presenza di molte logiche tra loro non riducibili, determinano una differenza qualitativa tra macro e micro-progettazione. Se un tempo si aveva una logica unica tra macro e micro, e la distinzione andava cercata sulla parcellizzazione dei contenuti o degli obiettivi, oggi macro- e micro-progettazione sono qualitativamente differenti.

Al centro della micro-progettazione si collocano le attività ovvero dispositivi articolati che coniugano in un percorso "integrated" (Bernstein, 1996) contenuti disciplinari, competenze e saperi ed esperienze degli studenti. Sono compiti autentici, attività di progetto, elaborazioni, attività di riflessione $\mathrm{e}$ riorganizzazione di frammenti, ovvero lavori difficilmente identificabili come "esercizi". La loro struttura articolata che prevede la presenza di interventi del docente, attività degli studenti, debriefing finale garantisce un dialogo, senza una 
riduzione all'identico, di materiali ed esperienze costruendo emergenze di senso. In tale direzione vanno le proposte teoriche del Conversational framework e delle Teaching Learning Activities (Laurillard, 2012), degli Episodi di apprendimento situato-EAS (Rivoltella, 2013), di DEPIT (Rossi, 2016).

Ben diversa è la logica della macro-progettazione. Se un tempo la macro era costituita da blocchi da cui derivava la micro-progettazione, con una logica simile a quella che Bernstein (1996) ha definito collection, oggi la macroprogettazione fornisce il senso globale, il significato di insieme, lo sfondo integratore, secondo una grammatica narrativa del percorso. Come suggerisce Bruner (1990) i soggetti trovano nella grammatica narrativa gli strumenti per raccontare e costruire la propria traiettoria personale e professionale. La classe è un soggetto sistemico e complesso che ha bisogno di raccontare e raccontarsi per identificare se stessa e le modalità con cui emerge in modo enattivo e nel tempo. Inoltre la macro-progettazione supporta le relazioni che esistono in una stessa disciplina tra sezioni in apparenza autonome, fa dialogare il diverso, superando le continue barriere che non sono presenti solo tra le discipline, ma anche tra settori della disciplina stessa: per la matematica, l'aritmetica, la geometria, l'algebra, l'analisi o per l'italiano l'analisi testuale, la letteratura, la grammatica e la sintassi. La macro-progettazione, in quanto narrazione, descrive anche lo sviluppo temporale ovvero annuale del percorso da fare oltre a fornire la metafora che dà il senso all'intero percorso.

\section{II curricolo trasposto - II visible design}

Se il livello macro presenta una traiettoria complessa non lineare, facilmente intuibile dallo studente, e il livello micro ha una struttura per attività, in cui confluiscono saperi ed esperienze, si attivano processi di approfondimento e riflessione, e si utilizzano materiali multimodali, lo studente va avviato a questo nuovo modello di studying. In tale direzione è stato sperimentato come efficace l'utilizzo in classe come mediatore tra docente e studenti di un artefatto che espliciti la struttura della macro- e micro-progettazione. Tale artefatto non è la progettazione che il docente ha predisposto secondo una propria logica e un proprio linguaggio, ma la trasposizione della stessa con una logica, un linguaggio e una grafica adeguati agli studenti. Tale artefatto permette di rendere gli studenti partecipi del percorso da sviluppare e della logica ad esso sottesa, supporto il loro orientamento nel processo e apre spazi di coprogettazione sul disegno macro e micro delle attività e vanno resi partecipi della logica globale.

Because of the greater focus in the research literature on methods seen as supporting constructivism, there are rather few recent studies on how 
to design lectures, written texts, websites, podcasts, or videos for learning. The few there are confine their advice to reiterating the conclusion from student learning research, that it is important to make the structure clear, and suggest signposting and organizing devices such as headings, graphics, advance organizers, repetition, and summaries (Biggs, 2003; Butcher, Davies, and Highton, 2006; Duffy, 2008; Fry, Ketteridge, and Marshall, 2003; Horgan, 2003; Mayer, 1999) (Laurillard, 2012, 150).

I pochi che esistono limitano i loro consigli al ribadire la conclusione della ricerca sull'apprendimento, secondo cui è importante creare una struttura chiara, suggerendo e organizzando espedienti come intestazioni, grafici, "organizzatori anticipati", ripetizioni e riassunti (Biggs, 2003; Butcher, Davies e Highton, 2006; Duffy, 2008; Fry, Ketteridge e Marshall, 2003; Horgan, 2003; Mayer, 1999).

La logica è quella dell'allineamento (Biggs, 2003, 27; Laurillard, 2012, 100). Se spesso per l'allineamento si è focalizzata l'attenzione sugli obiettivi, Laurillard precisa che in tale direzione non è sufficiente l'allineamento dei verbi sottesi all'obbiettivo, ma che lo studente deve essere consapevole dell'intero processo, grazie alla mediazione del docente.

The cycle of goal-knowledge-action-feedback-modulation constitutes what is learned and how. What the concept of alignment recognizes is that unless the teacher addresses the full cycle the intended learning outcome may not be achieved. It might be achieved, if the learner is able to build for themselves the alignment between intended learning outcomes $\longleftarrow \longrightarrow$ teaching-learning activity $\longleftrightarrow$ assessment, but the principle of alignment says that it is the teacher's role to ensure it (Laurillard, 2012, 96).

La conoscenza del senso e della struttura del percorso e dell'attività favorisce una partecipazione attiva e consapevole. Conoscere la struttura permette di anticipare e le neuro-scienze evidenziano il ruolo della previsione nei processi di conoscenza (Rivoltella, 2014). Per Berthoz (2009) l'anticipazione è un principio della semplessità. L'artefatto, frutto della trasposizione del curricolo, può essere visto come un elemento di semplessità in quanto permette agli studenti di gestire la complessità. Dalle sperimentazioni effettuate in alcune classi è emerso che sono proprio gli studenti con minori abilità a trarre maggior beneficio dalla presenza di un curricolo trasposto, in quanto la mediazione del curricolo fornisce a studenti culturalmente svantaggiati o abituati a modelli diversi di conoscere la logica dello studying e suggerisce l'approccio allo studio. In tal senso sembrano corretti i dubbi di alcuni autori (Parekh, 2017; Ladwig e McPherson, 2017) che reputano pericoloso supporre, spesso implicitamente, una relazione tra abilità, capacità cognitive e apprendimento. Nel caso in oggetto non è la semplificazione del processo, ma una diversa mediazione della struttura a migliorare gli apprendimenti. 
Nel passato, come più volte affermato nelle precedenti pagine, il curricolo, pur non presentato, era intuito dagli studenti perché era nota la logica dei vari corsi frequentati in quanto era la stessa con cui si erano misurati fin dai primi anni di studio: la logica lineare della linea del tempo (presente in discipline quali storia, storia della letteratura o storia della filosofia) o quella gerarchica dal particolare al generale (presente in discipline quali geometria, fisica, grammatica). Ma cosa accade se i percorsi divengono complessi e il senso della macro-progettazione non sia intuitivo e scontato, e le sessioni di lavoro siano dei processi di aggregazione complessa di esperienze, competenze e saperi disciplinari? In tal caso lo studente deve condividere il disegno globale e conoscerlo per poterlo possedere per poi partecipare a fasi di co-progettazione. La co-progettazione è un'esigenza in quanto il contenuto di molti percorsi è solo in parte precisato a priori e molto spesso viene definito in modo ricorsivo in itinere grazie all'interazione tra docenti e studenti, e tra studenti e studenti e all'apporto anche esperienziale degli stessi.

Da tutto ciò nasce l'esigenza di un terzo artefatto progettuale, dopo quelli del (1) Curricolo di istituto e della (2) macro- e micro-progettazione: (3) il curricolo trasposto ovvero una struttura a più livelli, macro e micro, da utilizzare in classe, da condividere con gli studenti e da arricchire con i materiali che si producono nelle attività presenti.

Il progetto PROPIT, attivato dal gruppo TINCTEC dell'Università di Macerata e, ora, il progetto europeo DEPIT ${ }^{12}$ hanno come finalità quella di valutare l'efficacia e la sostenibilità di tale terzo artefatto.

I progetti prevedono che la macro e la micro-progettazione divengano attraverso un processo di trasposizione didattica un artefatto digitale graficotestuale, ad esempio una mappa. Si avranno così le mappe che visualizzano contenuti trasposti della macro-progettazione e della micro-progettazione. L'artefatto essendo digitale garantisce che le due mappe siano tra loro connesse da link così che lo studente possa passare con un solo click dal macro al micro livello e viceversa, e che le stesse possono essere visualizzate in classe con una LIM e condivise dagli studenti anche su proprie device. Nella costruzione delle mappe particolare attenzione verrà posta al linguaggio utilizzato e alla struttura grafico-iconica. Gli artefatti, pertanto, tengono conto delle ricerche sul carico cognitivo, sulle problematiche dei BES e delle disabilità, sull'interculturalità.

${ }^{12}$ Il progetto DEPIT è stato approvato all'interno del programma Erasmus +. La partnership è costituita, oltre che dal Centro TincTec dell'Università di Macerata, dall'University College of London, dall'Universitad de Sivilla, dall'Università Cattolica del Sacro Cuore di Milano, dalle reti di scuole Crescendo (scuola capofila: IC di Grottamamre (AP) e Propit (scuola capofila: IC Torre di Pordenone), da Infofactory, dalle associazioni di docenti: ATEE e ADEE. Per maggiori informazioni si veda il sito: http://depit.eu. 
La presenza di un curricolo trasposto facilita processi inclusivi in quanto accompagna gli studenti nella comprensione del senso del percorso. Inoltre facilita la personalizzazione in quanto alcuni nodi della mappa sono in parallelo e prevedono attività da svolgere in parallelo da gruppi diversi della classe o in successione in funzione dei tempi di lavoro di ogni studente. Tali attività sono, pertanto, leggibili e gestibili direttamente dagli studenti, liberando così il docente dall'assegnare la consegna e permettendogli di dedicarsi a specifici gruppi presenti nella classe. In tal senso l'artefatto del curricolo trasposto va nella direzione di mediatizzare il mediatore, ovvero il docente (Damiano, 2013, 181).

Non solo la funzione dell'insegnante si esercita attraverso la regia del dispositivo di mediazione, ma diviene essa stessa una componente del dispositivo: essa stessa dispositivo (ivi, 283).

Nelle sperimentazioni fin qui effettuate il curricolo trasposto ha avuto non solo la funzione di artefatto progettuale. Essendo utilizzato in aula è stato arricchito con materiali significativi prodotti durante le attività, cosicché l'artefatto progettuale diviene anche in documentazione del percorso.

La valutazione delle esperienze effettuate in questi 5 anni hanno mostrato che dal punto di vista del docente realizzare una progettazione che rispecchi le attività, macro e micro, favorisce una implementazione della professionalità, una maggiore consapevolezza del progetto sotteso e del percorso. Inoltre la presenza di un canovaccio rende più agevole la gestione della classe e una chiara visione del processo. In tale direzione si conferma l'intuizione di Laurillard la quale ritiene che l'esplicitazione e la condivisione della progettazione favorisca la professionalizzazione dei docenti e

Teachers need the professional capacity to learn through collaboration, to build the knowledge of what it takes to teach. A step towards this would be to create the tools and the culture that enable teaching to be a design science ${ }^{13}$.

Dal punto di vista dello studente migliora l'orientamento, la consapevolezza e la visione globale del percorso annuale e una maggiore facilità a dialogare anche nella prospettiva macro della disciplina. Inoltre l'osservazione nelle classi sperimentali ha colto interventi di co-progettazione in cui gli studenti, in base alle loro conoscenze, hanno suggerito ampliamenti e connessioni nel percorso macro.

13 I docenti hanno bisogno della capacità professionale di imparare attraverso la collaborazione, di costruire la conoscenza di ciò che serve per insegnare. Un passo avanti in tale direzione è sicuramente creare gli strumenti e la cultura che consentano all'insegnamento di diventare una scienza della progettazione. 


\section{Conclusioni}

Le sperimentazioni degli ultimi anni effettuate dal gruppo TincTec per analizzare i curricoli di istituto e le macro- e micro-progettazioni, per far emergere le modalità con cui sono stati realizzati e per attivare il progetto PROPIT hanno confermato la necessità di un curricolo generativo e flessibile, di una distinzione tra macro e micro-progettazione esplicite e condivise, della presenza di un Curricolo trasposto.

Una pianificazione flessibile e dinamica, ma anche visibile è alla base di una didattica che risponda alle esigenze di una società e una cultura complessa e multi-prospettica e, contemporaneamente, favorisca il processo di professionalizzazione dei docenti che è alla base del processo precedente. Il nodo centrale è proprio la relazione tra processi produttivi (in cui il/i docente/i predispone/gono e gestisce/ono le attività didattiche) e processi costruttivi (in cui il docente riflette e lavora sulla propria professionalità). La ricorsività tra $\mathrm{i}$ due processi sorregge da un lato l'innovazione e l'avvio di pratiche coerenti con le esigenze culturali e sociali degli studenti, dall'altro richiede processi formativi (auto ed etero) nei quali si prende consapevolezza, si riflette e si mettono a punto i processi precedenti. In questa fase molte scuole hanno avviato processi di progettazione condivisa tra docenti che hanno sicuramente favorito una maggiore percezione della propria professionalità e una implementazione della stessa. Gli artefatti prodotti, in molti casi, risentono ancora dei modelli precedenti, ma le attività in atto stanno man mano creando la consapevolezza della poca efficacia di vecchi modelli e favoriscono la identificazione di strutture capaci di creare un ponte tra la progettualità di istituto e il lavoro nelle e con le classi.

\section{Riferimenti bibliografici}

Bernstein B. (1996). Pedagogy,Symbolic control and Identity. London: Taylor and Francis.

Berthoz A. (2009). La simplexité. Paris: Odile Jacob.

Biggs J. (2003). Teaching for quality learning at university. Buckingham: SRHE/OUP.

Bruner J. (1990). La ricerca del significato. Per una psicologia culturale. Torino: Bollati-Boringhieri.

Joannert P. (2011). Curriculum, entre modèle rationnel et irrationalité des sociétés. Revue Interntionale d'éducation. 56, 135-145.

Jonnaert P., Van der Borght C. (2009). Créer des conditions d'apprentissage. Un cadre de référence socioconstructiviste pour la formation didactique des enseignants. Paris/Bruxelles: De Boeck-Université.

Ladwig J., McPherson A. (2017). The anatomy of ability. Curriculum Inquiry. 47, 4, 344-362. 
Laurillard D. (2012). Teaching as design science. London: Roudledge.

Parekh G. (2017). The tyranny of "ability". Curriculum Inquiry. 47, 4, 337-343.

Rivoltella P.C. (2013). Fare didattica con gli EAS. Episodi di Apprendimento Situati. Brescia: La Scuola.

Rivoltella P.C. (2014). La previsione. Neuroscienze, apprendimento, didattica. Brescia: La Scuola.

Rossi P.G. (2016). Microprogettazione. Milano: FrancoAngeli.

Stenhouse L. (1991). Dalla scuola del programma alla scuola del curricolo. Roma: Armando.

Vygotskij L.S. (1954). Pensiero e linguaggio. Firenze: Giunti-Barbera. 